\title{
A HEURISTIC REVIEW OF SOME RUIN THEORY RESULTS
}

\author{
By G. C. TAYLOR
}

E. S. Knight \& Co., Sydney

\begin{abstract}
The paper deals with the renewal equation governing the infinite-time ruin probability. It is emphasized as intended to be no more than a pleasant ramble through a few scattered results. An interesting connection between ruin probability and a recursion formula for computation of the aggregate claims distribution is noted and discussed. The relation between danger of the claim size distribution and ruin probability is reexamined in the light of some recent results on stochastic dominance. Finally, suggestions are made as to the way in which the formula for ruin probability leads easily to conclusions about the effect on that probability of the long-tailedness of the claim size distribution. Stable distributions, in particular, are examined.
\end{abstract}

\section{KEYWORDS}

Ruin probability, recursive computation of aggregate claims distribution, danger of claim size distribution, stable claim size distributions.

\section{INTRODUCTION}

Subsequent sections of this paper examine various aspects of ruin theory from viewpoints which are very much heuristic. They contain very little original material. Rather are they concerned with modes of thought which may be applied to the various aspects of ruin theory considered in order to yield a better understanding of them than is obtained by concentration on the mathematical detail. From this point of view, the paper possibly has some didactic value and indicates lines of thought which can lead to valuable conjectures-these latter to be tested subsequently of course by rigorous mathematics.

Of course, ruin theory has been with us for the best part of the present century, dating back to LUNDBERG (1909). However, the recent past has brought a number of new techniques which facilitate manipulation of the theory, or indeed coherent and suggestive thought on the subject. A number of the references given at the end of the paper are quite modern, indicating the extent to which these new techniques have been developing.

The particular aspect of ruin theory which has been selected to form the subject of this paper is the renewal equation governing the infinite-time ruin probability. The form of this equation is set out in Section 2. Subsequent sections derive from

\footnotetext{
* Invited address to the Astin meeting of the 22nd International Congress of Actuaries, Sydney, 26 October 1984.
}

ASTIN BULLETIN Vol. 15, No. 2 
that equation a few results on computation of ruin probabilities (Section 3 ), the effect of danger of the claim size distribution (Section 4), and the effect of long and short tailed claim size distributions (Section 5).

Undoubtedly, other topics could have been covered. However, it is to be emphasised that the present paper is intended to be no more than a pleasant, and slightly different, ramble through a few scattered results.

\section{THE BASIC EXPRESSION FOR RUIN PROBABILITY}

Consider the classical ruin process. That is, an initial reserve of $x \geqslant 0$ is increased by premium income at the rate of $c$ per unit time and decreased at random epochs by claim payments.

These claims are generated by a Poisson process with a mean density of 1 claim per unit time. The sizes of the claims are i.i.d. with d.f. $(B \cdot)$ whose mean is 1 .

Write $c=1+\eta$, where $\eta$ is the safety loading per unit of risk premium.

Define

$$
h(y)=[1-B(y)] / c
$$

and

$$
F(x)=\int_{0}^{x} h(y) d y
$$

Note that $F(\cdot)$ is a defective d.f. It is possible to rewrite it as:

$$
F(x)=G(x) / c
$$

where

$$
G(x)=\int_{0}^{x} g(y) d y
$$

with

$$
g(y)=1-B(y)=\operatorname{ch}(y) .
$$

Since the mean associated with $B(\cdot)$ is unity, $G$ is a non-defective d.f.

Now, let $\phi(x)$ denote the probability that the free reserve generated by the initial reserve $x$ and the claims process described above remains non-negative at all times, i.e., $\phi(x)$ is the infinite-time probability of non-ruin given initial reserve $x$.

As any of the standard texts on ruin theory shows (BüHLMANN, 1970; FELLER, 1966; GERBER, 1979; SEAL, 1969), $\phi(x)$ satisfies a renewal equation:

$$
\phi(x)=\eta / c+\int_{0}^{x} \phi(x-y) h(y) d y .
$$

Finally, define the infinite-time probability of ruin given initial reserve $x$ :

$$
\psi(x)=1-\phi(x)
$$


Exact and approximate solutions to equation (2.4) appear in various forms (again see the standard texts). The particular form of the solution with which the present exposition will concern itself is the following:

$$
\phi(x)=(\eta / c) U(x)
$$

where

$$
U(x)=\sum_{n=0}^{\infty} F^{n *}(x),
$$

the $F^{n *}(\cdot)$ being defined in the usual way as a convolution:

$$
\begin{aligned}
F^{n *}(x) & =\int_{0}^{x} F^{(n-1) *}(x-y) d F(y) \\
& =\int_{0}^{x} F^{(n-1) *}(x-y) h(y) d y .
\end{aligned}
$$

By (2.5) and (2.6), the probability of non-ruin $\phi(x)$ involves a sum of d.f.'s. It is inconvenient for some purposes that these d.f.'s are defective. They may be converted to non-defective by means of (2.2) and (2.3). Thus, (2.6) and (2.7) may be replaced by the following:

$$
U(x)=\sum_{n=0}^{\infty} c^{-n} G^{n *}(x),
$$

with

$$
\begin{aligned}
G^{n *}(x) & =\int_{0}^{x} G^{(n-1) *}(x-y) d G(y) \\
& =\int_{0}^{x} G^{(n-1) *}(x-y) g(y) d y .
\end{aligned}
$$

3. AN AGGREGATE CLAIMS AMOUNT REPRESENTATION OF SURVIVAL PROBABILITY

\subsection{The Representation}

If we write $p=1 /(1+\eta)$, then (2.8) takes the form:

$$
U(x)=(1+\eta) \eta^{-1} \sum_{n=0}^{\infty}(1-p) p^{n} G^{n *}(x),
$$

and, by $(2.5)$,

$$
\phi(x)=\sum_{n=0}^{\infty}(1-p) p^{n} G^{n *}(x)
$$

This is an interesting representation of the survival probability because it can be recognised as the d.f. of aggregate claims when the number of claims has a geometric distribution (parameter $p$ ) and claim size has d.f. $G(\cdot)$ 
This representation can be found in Bowers, Gerber, Hickman, Jones and NesBitT (1982, Section 12.6), though their development of it is a little different from here. It is repeated in PANJER (1984).

It follows that any algorithm available for the evaluation of the aggregate claims distribution (subject to geometric claim frequency) can be used immediately to evaluate ruin probability.

\subsection{A Connection with Panjer's Recursion}

Evaluation of the aggregate claims distribution is precisely the purpose of Panjer's recursion (PANJer, 1981; Sundt and Jewell, 1981). This recursion can be applied to various claim frequency distributions of which the geometric distribution is one.

Panjer's formula is:

$$
g(x)=p_{1} f(x)+\int_{0}^{x}(a+b y / x) f(y) g(x-y) d y, \quad x>0,
$$

where $g(\cdot)$ is the (continuous) p.d.f. of aggregate claims; $p_{n}$ is the probability of $n$ claims in the time period under consideration; $f(\cdot)$ is the p.d.f. of individual claim size; and $a, b$ are parameters characterizing the claim frequency distribution which must satisfy:

$$
p_{n}=p_{n-1}(a+b / n) .
$$

For the geometric claim frequency distribution appearing in Section 3.1, $a=p$, $b=0$. Moreover, in the application of recursion (3.2.1) to (3.1.1) it is necessary to replace $f$ by $g$, the p.d.f. associated with $G$ (N.B. this $g$ is not the same as that appearing in (3.2.1); and $g$ by $\phi^{\prime}$, the derivative of $\phi$. In this last replacement, $\phi^{\prime}$ plays the role of a notional p.d.f. associated with $\phi$ when the latter is viewed as a d.f.

With these replacements, (3.2.1) becomes:

$$
\phi^{\prime}(x)=(1-p) p g(x)+p \int_{0}^{x} g(y) \phi^{\prime}(x-y) d y .
$$

\subsection{Recovery of the Renewal Equation from Panjer's Recursion}

The particular application (3.2.3) of Panjer's recursion provides $\phi^{\prime}$ rather than $\phi$. The survival probability may be obtained by integrating (3.2.3). First, (3.2.3) is rewritten using the fact that $g(y)=\operatorname{ch}(y)$, by (2.3);

$$
\begin{aligned}
\phi^{\prime}(x) & =(1-p) h(x)+\int_{0}^{x} h(y) \phi^{\prime}(x-y) d y \quad[p c=1] \\
& =(d / d x) \int_{0}^{x} h(y) \phi(x-y) d y,
\end{aligned}
$$


where the last step has used the fact that $\phi(0)=\eta / c=1-p$. Then

$$
\phi(x)=\eta / c+\int_{0}^{x} h(y) \phi(x-y) d y,
$$

again using the fact that $\phi(0)=\eta / c$.

Thus, the basic renewal equation (2.4) has been recovered from Panjer's recursion (3.2.3).

Though a little academic, it is perhaps fascinating to note that the above argument could have been applied in reverse, beginning with the renewal equation (2.4). From this could have been derived (3.2.3), and hence (3.2.1) for the special case in which:

(i) claim frequency follows a geometric distribution;

(ii) d.f. of aggregate claims amount is the same as some survival probability $\phi(\cdot)$.

In other words, Panjer's recursion formula for aggregate claims distribution might easily have arisen as a conjecture derived from the basic renewal equation of ruin theory.

\subsection{Computation of Ruin Probability}

As noted in Section 3.1, algorithms available for the evaluation of aggregate claims amount distribution may be readily applied to the evaluation of ruin probability. Formula (3.2.3) provides one such example.

Practical application of such techniques in general circumstances require that formulas like (3.2.3) be discretized. The discrete version of Panjer's recursion (3.2.1) is provided by PANJER (1981, p. 25):

$$
g_{i}=p \sum_{j=1}^{i} f_{j} g_{i-j}
$$

where $g_{i}, f_{i}$ are the same p.d.f.'s as in Section 3.2 except that the distributions are now discrete with mass points $0, h, 2 h, \ldots, i h, \ldots$, for some step $h>0$.

The recursion (3.4.1) may be adapted to the ruin probability case in the same way as (3.2.1) yielded (3.2.3):

$$
(d \phi)_{i}=p \sum_{j=1}^{i} g_{j}(d \phi)_{i-j}
$$

where $g_{j}$ now represents the discretized version of the p.d.f. $g(\cdot)$ defined by $(2.3)$ and $(d \phi)_{i}$ is the increase in the function $\phi(x)$ over the interval $\left(\left(i-\frac{1}{2}\right) h,\left(i+\frac{1}{2}\right) h\right)$. Similarly, we adopt $(d \phi)_{0}=\phi(0)=1-p$.

Shortly after circulation of the announcement of this lecture, Prof. H. H. Panjer advised that he had been working along similar lines. A formula corresponding to (3.4.2) (in fact, a more refined version) can be found in PANJER (1984).

Similar procedures have also appeared recently in the work of GoOvAERTS and DE VYLDER (1984a). 
It should be noted that, by (2.3), the p.d.f. $g(\cdot)$ always exists and so the corresponding distribution cannot in fact be discrete. The discretization (3.4.2) is necessarily, therefore, an approximation only.

Some further difficulties occur in relation to the existence of some mass at the zero point under the "distribution function" $\phi(\cdot)$. As this paper is concerned only with broad ideas, these difficulties are not pursued. The details of their treatment are provided by PANJER (1984).

\subsection{Lundberg-Type Approximation to Ruin Probability}

Suppose that the distribution associated with $g(\cdot)$ is approximated by a distribution of a finite random variable. This is done by assuming that

$$
g_{j}=0 \quad \text { for } j>J \text {. }
$$

Then (3.4.2) reduces to:

$$
(d \phi)_{i}=\sum_{j=1}^{J}\left(p g_{j}\right)(d \phi)_{i-j} \quad \text { when } i \geqslant J .
$$

This is a linear recurrence relation in the $(d \phi)_{i}$ with constant coefficients $p g_{j}$. The asymptotic solution of such a system for large $i$ is well-known. It takes the form:

$$
(d \phi)_{i}=\text { const. } \times \alpha^{i}+o\left(\alpha^{i}\right),
$$

for some positive constant $\alpha$.

It follows that

$$
\begin{aligned}
\psi(i h) & =\sum_{j=i+1}^{\infty}(d \phi)_{i} \\
& =\text { const. } \times \alpha^{i}+o\left(\alpha^{i}\right),
\end{aligned}
$$

i.e., putting $x=i h, \alpha=\exp (-R h), R$ const. $>0$ (provided $p<1$ ),

$$
\psi(x) \sim C \exp (-R x) \text { for } x \rightarrow \infty,
$$

where $C$ is a constant $>0$.

The result (3.5.3) is recognised as the usual ruin probability approximation of the Lundberg type.

\subsection{Finite-Time Ruin Probability}

The great bulk of this paper is concerned with the infinite time probability of ruin $\psi(x)$. However, there is one very simple application of Panjer's results to the finite-time ruin probability.

Let $\phi(x, t)$ denote the probability of survival over the time interval $[0, t]$ when the initial free reserve is $x$. Thus, $\phi(x)=\phi(x, \infty)$.

SEAL (1969, p. 98) shows that:

$$
\phi(0, t)=\eta / c+(c t)^{-1} \int_{c t}^{\infty}[1-P(y, t)] d y,
$$


where $P(y, t)$ is the probability that aggregate claims in the time interval $[0, t]$ do not exceed $y$.

Note that $P(\cdot, t)$ may be evaluated by means of Panjer's recursion. To the extent that this recursion simplifies the computation of the distribution of aggregate claims amount, it also simplifies computation of $\phi(0, t)$.

\section{RUIN PROBABILITY AND DANGER OF THE CLAIM SIZE DISTRIBUTION}

The concept of danger of a claim size distribution is introduced after the manner of Bühlmann, Gagliardi, Gerber and Straub (1977). Thus, it is said that one claim size distribution with d.f. $B_{1}$ is more dangerous than another with d.f. $B_{2}$ if:

(i) $\infty>\mu_{1} \geqslant \mu_{2}$, where $\mu_{1}, \mu_{2}$ are the means of $B_{1}, B_{2}$ respectively;

(ii) there exists a constant $a$ such that:

$$
\begin{array}{ll}
B_{1}(x) \geqslant B_{2}(x) & \text { for } x<a ; \\
B_{1}(x) \leqslant B_{2}(x) & \text { for } x \geqslant a .
\end{array}
$$

The following proposition, proved by BühlmanN, Gerber, Gagliardi and STRAUB $(1977$, p. 80 ) establishes a connection between danger of a distribution and stop-loss premium.

Proposition 4.1. If $B_{1}, B_{2}$ are d.f.' $s$ and $B_{1}$ is more dangerous than $B_{2}$, then, for each real $t$ :

$$
\int_{t}^{\infty}(x-t) d B_{1}(x) \geqslant \int_{t}^{\infty}(x-t) d B_{2}(x) .
$$

i.e. for any given retention, $B_{1}$ generates stop-loss premiums at least as great as $B_{2}$.

It is convenient to introduce the terminology of GoovaerTs, DE VyLder and HAEZENDONCK (1984), who refer to (4.1) as second-degree stop-loss dominance of $B_{1}$ over $B_{2}$. The general definition of stop-loss dominance of course encompasses $n$th degree dominance. Goovaerts, de Vylder and Haezendonck establish relations between this type of dominance and stochastic dominance.

Present interest is in only first-order stochastic domiance, defined as follows. A d.f. $B_{1}$ is said to have first-order stochastic dominance over d.f. $B_{2}$ if $B_{1}(x) \leqslant B_{2}(x)$ for all $x$.

Also of interest for present purposes are the following propositions (Goovaerts, De Vylder and Haezendonck, 1984, p. 308).

PRoposition 4.2. Stochastic dominance of nth degree is preserved under mixing of distributions.

Proposition 4.3. Stochastic dominance of $n$th degree is preserved under convolution of distributions. 
Now consider how these results bear upon the ruin probability given by (2.5) and $(2.8)$. It is seen that survival probability $\phi(x)$ is obtained by:

(i) convolution of the d.f. $G(\cdot)$;

(ii) mixing of the resulting convolutions.

Now let $G_{i}, U_{i}, \phi_{i}$ be associated with $B_{i}, i=1,2$, through (2.3), (2.8) and (2.5) respectively. Note that the stop-loss dominance (4.1) may be written equivalently as:

$$
\int_{t}^{\infty}\left[1-B_{1}(x)\right] d x \geqslant \int_{t}^{\infty}\left[1-B_{2}(x)\right] d x
$$

i.e.,

$$
\mu_{1}-\int_{0}^{t}\left[1-B_{1}(x)\right] d x \geqslant \mu_{2}-\int_{0}^{t}\left[1-B_{2}(x)\right] d x,
$$

when $\mu_{1}, \mu_{2}<\infty$, and assuming that $B_{1}(0)=B_{2}(0)=0$ (i.e., positive claim sizes). Thus, if

$$
\mu_{1}=\mu_{2},
$$

then, by $(2.3),(4.1)$ is equivalent to

$$
G_{1}(x) \leqslant G_{2}(x) \text { for each } x,
$$

i.e. $G_{1}$ is first-degree stochastically dominant over $G_{2}$.

The above results may be combined to yield the following:

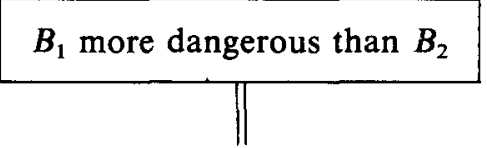

$$
\text { by (4.3) }
$$

(by definition, $\mu_{1}=\mu_{2}=1$ )

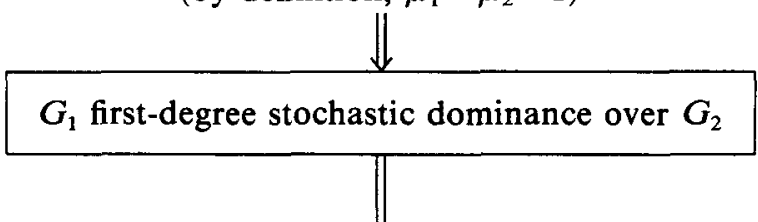

Propositions 4.2 and 4.3

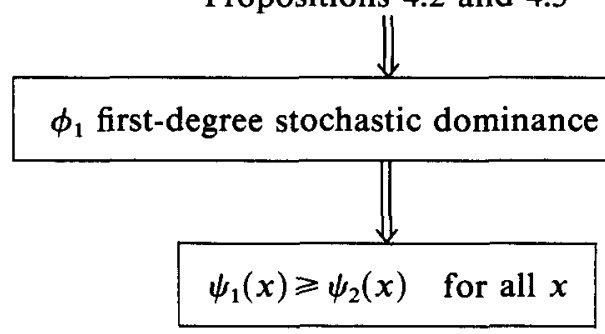


Thus, the following result, originally proved by TAYLOR (1976, pp. 204-205) is recovered.

Proposition 4.4. If one individual claim size distribution $B_{1}$ is more dangerous than another $B_{2}$, then the associated infinite-time ruin probabilities are related as follows:

$$
\psi_{1}(x) \geqslant \psi_{2}(x) \text { for all } x .
$$

Moreover, it may be checked through the above proof that equality between $\psi_{1}(x)$, $\psi_{2}(x)$ for all $x$ occurs only if $B_{1}, B_{2}$ are identical a.e.

Taylor obtained this result by means of integral inequalities. Here the same result has been obtained by much simpler and more direct methods. Rather similar methods are used by DE VYLDER and GoovaERTs (1984b, Theorem 2) to establish the same result.

As a final remark, brief reference is made to the discussion in Sections 4 and 5 of GoOVAERTS and DE VYLDER (1984b). It is pointed out there that the result of Proposition 4.4 can be reversed in the case of finite-time ruin probabilities.

Indeed, it follows immediately from (3.6.1) that:

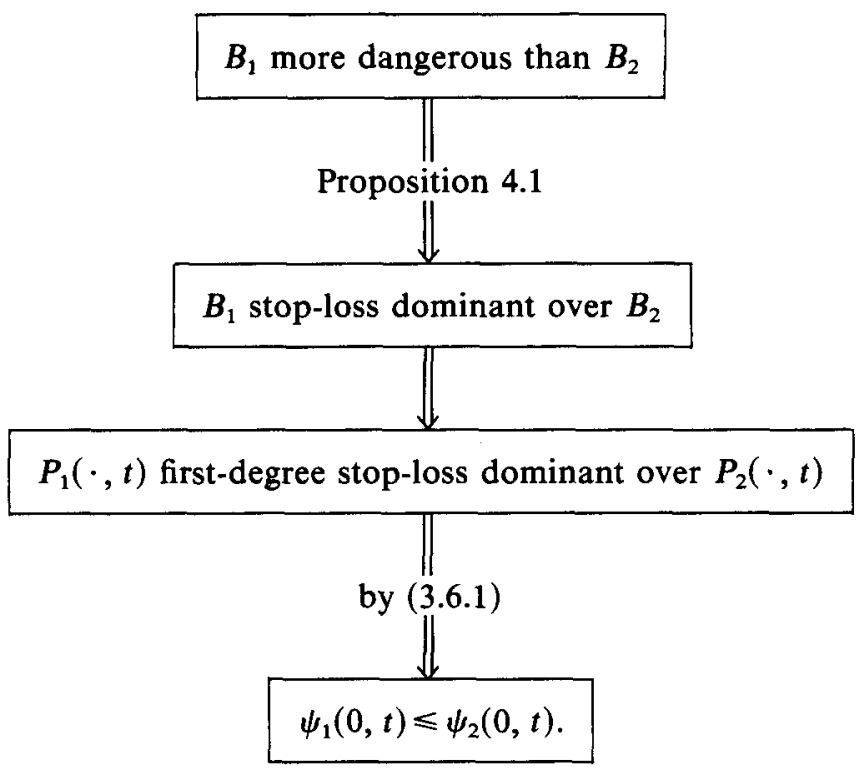

In this reasoning $P_{i}(\cdot, t)$ denotes $P(\cdot, t)$ of (3.6.1) with $B(\cdot)$ replaced by $B_{i}(\cdot), i=$ 1,2 . The first step in the chain of reasoning is easily derivable from results of GoOvaERTS and DE VyLder (1984a, Theorems 6 and 7) on preservation of stop-loss dominance under mixing and convolution.

The above result is extended somewhat by GoOVAERTS and DE VYLDER (1984b, especially Theorem 4 and the diagram in Section 5). 
It is interesting to let $t \rightarrow \infty$ in the last result, and compare with Proposition 4.4. It then follows that

$$
\psi_{1}(0)=\psi_{2}(0)
$$

regardless of the respective dangers of $B_{1}$ and $B_{2}$.

This result is consistent with the well-known general result:

$$
\psi(0)=1-\eta / c .
$$

\section{INFLUENCE OF CLAIM SIZE DISTRIBUTION ON PROBABILITY OF RUIN}

\subsection{General}

This section is intended to be no more than heuristic. The issues to be examined briefly are:

(i) the reasons why $\psi(x)$, as a function of $x$, differs so greatly according to whether the distribution of individual claim size is long or short tailed;

(ii) the meaning of the terms long and short tailed in (i);

(iii) in the short tailed case, the insensitivity of the function $\psi(x)$ to the detailed shape of $B(\cdot)$.

To begin with it will be helpful to write $\bar{G}^{n *}(\cdot)$ as the d.f. of the mean of $n$ drawings from the d.f. $G(\cdot)$, whereas $G^{n *}$ is the d.f. of the sum of the $n$ drawings. Thus,

$$
\bar{G}^{n *}(x)=G^{n *}(n x)
$$

Combination of (2.8) and (5.1.1) yields:

$$
U(x)=\sum_{n=0}^{\infty} c^{-n} \bar{G}^{n *}(x / n) .
$$

The two expressions for $U(x),(2.8)$ and (5.1.2), show that the properties of $\psi(x)$ are determined by the properties of sums of drawings from the d.f. $G(\cdot)$, or on the alternative view by the properties of means of drawings from $G(\cdot)$.

\subsection{A Heuristic Argument}

The final observation of Section 5.1 leads one naturally to consider application of the central limit theorem.

We shall return shortly to the question of applicability of this theorem, but suppose for the moment it can be applied to $\bar{G}^{n *}(x / n)$ in (5.1.2). Then, as $n \rightarrow \infty$,

$$
\bar{G}^{n *}(x / n) \rightarrow \Phi\left(x / n ; \mu, \sigma^{2} / n\right)
$$

the probability that a normal variate with mean $\mu$ and variance $\sigma^{2} / n$ assumes a value of $x / n$ or less, $\mu$ and $\sigma^{2}$ being the mean and variance of $G(\cdot)$. The convergence in (5.2.1) is convergence in measure. 
Note also that (5.1.2) may be rewritten as:

$$
U(x)=c / \eta-\sum_{n=0}^{\infty} c^{-n}\left[1-\bar{G}^{n *}(x / n)\right]
$$

and consider large $x$.

For small values of $n$, the convergence in (5.2.1) does not take effect, but the summands in (5.2.2) are small anyway because of the largeness of $x$. As $n$ becomes large, (5.2.1) takes effect so that (5.2.2) gives $U(x)$ approximately as:

$$
c / \eta-\sum_{n=0}^{\infty} c^{-n}\left[1-\Phi\left(x / n ; \mu, \sigma^{2} / n\right)\right] .
$$

This is very much a heuristic result. There are two aspects of it which have not been treated with care.

Firstly, no attention has yet been given to the validity of the convergence (5.2.1). While quite mild conditions on $G(\cdot)$ enable the conclusion that $\bar{G}^{n *}\left(x_{n}\right) \rightarrow$ $\Phi\left(x_{n} ; \mu, \sigma^{2} / n\right), x_{n}=\mu+x \sigma / \sqrt{n}$, as $n \rightarrow \infty$, relation (5.2.1) is another matter entirely. For in this latter case, the value of the argument of $\bar{G}^{n *}(\cdot)$ at which convergence is sought is varying with $n$ in another way; and in fact varying further into the left tail of $\bar{G}^{n *}(\cdot)$.

Secondly, proper deduction of (5.2.3) from (5.2.1) and (5.2.2) would require some investigation of effect of accumulating (by summation over $n$ ) the errors of approximation of $\bar{G}^{n *}(x / n)$ by $\Phi\left(x / n ; \mu, \sigma^{2} / n\right)$.

Neither of these matters has been treated above. Nor will any complete treatment be attempted. However, it will become apparent in Section 5.4 that (5.2.1) is not valid for certain distributions $G(\cdot)$ which are long tailed in the sense described there. It will also appear in Section 5.3 that a rigorous evaluation of (5.2.2) in the case of certain short tailed distributions (in the sense defined there) leads to results broadly compatible with (5.2.3).

Thus it appears that the validity of (5.2.3) as a rough approximation to $U(x)$ for large $x$ depends on the d.f. $G(\cdot)$ being sufficiently short tailed. The meaning of "short tailed" will be considered further in Section 5.3. If $G(\cdot)$ is not sufficiently short tailed, then the appearance of $\Phi$ in the approximation (5.2.3) is not justified, and one might suspect that $U(\cdot)$, and therefore $\psi(\cdot)$ undergoes radical change as $G(\cdot)$ moves from distributions which are short tailed to those which are not. It will be seen in Sections 5.3 and 5.4 that this is indeed the case.

\subsection{Ruin Probability for Short Tailed Claim Size Distributions}

For the moment, (5.2.3) continues to be taken on trust as an approximation to $U(x)$ for large $x$. Then, by (2.5),

$$
\psi(x)=1-\phi(x) \sim(\eta / c) \sum_{n=0}^{\infty} c^{-n}\left[1-\Phi\left(x / n ; \mu, \sigma^{2} / n\right)\right] .
$$

This expression is not particularly easy to evaluate, but it does convey one significant fact about $\psi(x)$ for large $x$. Since the right side of (5.3.1) is determined 
by just $\eta, \mu, \sigma^{2}$, there must be an approximation to $\psi(x)$ also dependent on just these quantities. By the definition of $G(\cdot)$ in Section 2 ,

$$
\mu=\alpha_{2} / 2, \quad \sigma^{2}=\alpha_{3} / 3-\left(\alpha_{2} / 2\right)^{2},
$$

where $\alpha_{k}$ denotes the $k$ th moment about the origin of claim size d.f. $B(\cdot)$; and where (5.3.2) has been computed on the assumption that $B(0)=0$, i.e., claim sizes are positive. Note that, by assumption, $\alpha_{1}=1$.

The conclusion from (5.3.2) and the paragraph preceding it is that there is a reasonable approximation to $\psi(x)$ for large $x$ depending on just $\eta$ and the first three moments of the claim size distribution.

This will now be shown consistent with the Lundberg type of approximation (e.g., SeAL, 1969, p. 131, who also cites LundBerG, 1909, 1926):

$$
\psi(x) \sim \text { const. } \times e^{-R x},
$$

where the "adjustment coefficient" $R>0$ is the solution of the characteristic equation:

$$
\int_{0}^{\infty} e^{R x}[1-B(y)] d y=1+\eta
$$

This solution has been shown (TAYLOR, 1974, p. 12) to take the form:

$$
R=\frac{2_{\eta}}{\alpha_{2}}-\frac{\alpha_{3}}{6}\left(\frac{2_{\eta}}{\alpha_{2}}\right)^{2}+O\left(\frac{2_{\eta}}{\alpha_{2}}\right)^{3} .
$$

Similar results have been obtained by DE VYLDER (1978).

It is clear that, as predicted, there is an approximation to $\psi(x)$ for large $x$ (namely (5.3.3)) which is essentially determined by $\eta$ and the first three moments of claim size distribution.

This observation tends to break down if $\eta$ becomes too large in (5.3.5). This is reasonable because, for large $\eta$ (i.e., large $c$ ), the higher order terms in (5.2.2) assume reduced importance. Correspondingly, there is increased emphasis on the lower order terms where the approximation (5.2.1) is poor. Hence (5.2.3) cannot be expected to lead to a good approximation to $\psi(x)$.

Moreover, it must be noted that the adjustment coefficient $R$ exists, i.e., (5.3.4) has a solution, only if the moment generating function of $[1-B(y)]$ as a p.d.f. exists. For positive claim sizes, this is the same as requiring that the m.g.f. of the claim size distribution exist. This in turn is the same as requiring that the tail of the claim size distribution converge to zero at least as rapidly as some negative exponential distribution.

If this last requirement be taken as defining short tailed distributions, then it is seen that:

(i) short tailed claim size distributions yield ruin probabilities quite compatible with the heuristic result of Section 5.2;

(ii) such ruin probabilities $\psi(x)$ may be approximated, for large $x$ and $\eta$ not too large, by an expression depending on just $\eta$ and the first three moments of the (short tailed) claim size distribution. 


\subsection{Ruin Probability for Long Tailed Claim Size Distributions}

\subsubsection{General}

Since Section 5.3 considered short tailed claim size distributions as those converging exponentially to zero, attention is now turned to others.

Recall from Section 5.1 that the properties of $\psi(x)$ are determined by the properties of sums of drawings from the claim size distribution. This suggests examining two classes of distribution:

(i) the subexponential class;

(ii) the stable distributions.

\subsubsection{Claim Size Distributions Related to the Subexponential Class}

These are dealt with in the ruin theory context by EMBrechTs and VERAVERBEKE (1982). A d.f. $G(\cdot)$ on $[0, \infty)$ is said to be subexponential if

$$
\lim _{x \rightarrow \infty} \frac{1+G^{2 *}(x)}{1-G(x)}=2 .
$$

EMbrechts and VeraVerbeke (1982, p. 62) point out that the m.g.f. of any member of the subexponential class does not converge. Thus, such distributions are indeed long tailed in the sense of Section 5.3. The cited authors point out that the lognormal and Pareto distributions lie in this class.

As noted by EMBRECHTS and VeraVerbeke (1982, p. 62),

$$
1-G^{n *}(x) \sim n[1-G(x)] \text { for large } x .
$$

This has immediate consequences for (2.8). If this latter is rewritten as:

$$
U(x)=c / \eta-\sum_{n=0}^{\infty} c^{-n}\left[1-G^{n *}(x)\right],
$$

for $G(\cdot)$ subexponential, then substitution of (5.4.2.1) yields:

$$
\begin{aligned}
& U(x) \sim c / \eta-\sum_{n=0}^{\infty} n c^{-n}[1-G(x)] \text { for large } x \\
& \begin{aligned}
\psi(x) & \sim \eta / c\left[\sum_{n=0}^{\infty} n c^{-n}\right][1-G(x)] \\
& =\frac{1}{\eta}[1-G(x)] \text { for large } x .
\end{aligned}
\end{aligned}
$$

Once again, quite inadequate attention has been given to convergence questions in the cavalier substitution of approximation (5.4.2.1) under the infinite sum of (5.4.2.2). Hence (5.4.2.3) can be viewed as no more than a heuristic result.

Nevertheless, at the heuristic level and by the very simple procedure illustrated above, we have obtained precisely one of the main results of EMBRECHTS and 
Veraverbeke (1982, Theorem 4.6), viz. if $G(\cdot)$ as defined in Section 2 is in the subexponential class, then (5.4.2.3) holds.

Note that, for large $x, \psi(x)$ then follows the complementary d.f. of $G(\cdot)$. This stands in contrast with the situation for short tailed claim size distributions whose asymptotic ruin probabilities are essentially characterized by only $\eta$ and the low order moments of the claim size distribution. This is a manifestation of the phenomenon, foreshadowed at the end of Section 5.2, whereby $(\cdot)$ undergoes a radical change as claim size distribution changes from short tailed to long tailed.

It is interesting to compare the approximation (5.4.2.3) with the inequality derived by BroeckX, Goovaerts and De Vylder (1984):

$$
\psi(x) \geqslant \frac{1}{\eta}[1-G(x)] \beta(x)
$$

with $\beta(x) \rightarrow 1$ as $x \rightarrow \infty$. This bound on ruin probability applies to any claim size distribution.

\subsubsection{Claim Size Distributions Related to the Stable Distributions}

Recall that the d.f. $G(\cdot)$ defined in Section 2 is said to be stable if:

$$
G^{n *}(x)=G\left(a_{n}^{-1}\left(x-b_{n}\right)\right),
$$

for some centralizing constants $b_{n}>0$ and norming constants $a_{n}>0$.

A useful, somewhat weaker, concept is the following. The d.f. $G(\cdot)$ is said to belong to the domain of attraction of another d.f. $H(\cdot)$ if there exist constants $a_{n}, b_{n}>0$, such that:

$$
G^{n *}(x) \rightarrow H\left(a_{n}^{-1}\left(x-b_{n}\right)\right) \text { as } n \rightarrow \infty .
$$

Only a stable distribution can have a domain of attraction (FELLER, 1966, p. 576).

A stable distribution $H(\cdot)$ is characterized by an exponent $0<\alpha \leqslant 2$ which has the following significance (FELLER, 1966, p. 576):

$$
1-H(x) \sim \text { const. }[(2-\alpha) / \alpha] x^{-\alpha} \text { for } x \rightarrow \infty .
$$

Also, if $G(\cdot)$ is in the domain of attraction of $H(\cdot)$, then $G(\cdot)$ has the same tail:

$$
1-G(x) \sim \text { const. }[(2-\alpha) / \alpha] x^{-\alpha} \text { for } x \rightarrow \infty .
$$

The norming constants are related to this exponent (FELler, 1966, p. 170):

$$
a_{n}=n^{1 / \alpha} \text {. }
$$

Moreover, it is known (FEller, 1966, p. 171) that $b_{n}=b\left(a_{n}-n\right)$ for some constant $b$.

Now suppose that the claim size distribution is such that $G(\cdot)$ lies in the domain of attraction of a stable d.f. $H(\cdot)$. Substitution of (5.4.3.3) and (5.4.3.5) in (5.4.3.2) gives, for large $x$ :

$$
\begin{aligned}
1-G^{n *}(x) & \rightarrow \text { const. }[(2-\alpha) / \alpha]\left[n^{-1 / \alpha}\left(x-b_{n}\right)\right]^{-\alpha} \\
& =\text { const. }[(2-\alpha) / \alpha] n x^{-\alpha}\left(1-b_{n} / x\right)^{-\alpha} \text { as } n \rightarrow \infty
\end{aligned}
$$


Note the tail form (5.4.3.4) of $G(\cdot)$, and hence of $G^{n *}(\cdot)$ since $G$ in the domain of attraction of $H$ implies the same $G^{n *}$. Hence the requirement of large $n$ may be dropped from (5.4.3.6), i.e.,

$$
1-G^{n *}(x) \sim[(2-\alpha) / \alpha] \times n[1-G(x)] \text { for large } x .
$$

Thus, for $0<\alpha<2$, self-convolution of a d.f. in the domain of attraction of a stable d.f. produces the same result (i.e., (5.4.2.1)) as obtained with a subexponential d.f.

Now note that the right side of (5.4.3.7) vanishes when $\alpha=2$, which case corresponds to the case of normal, and therefore short tailed, $H(\cdot)$. Thus, (5.4.3.7) translates as:

$$
\psi(x) \sim \text { const. } \times[1-G(x)] \text { for large } x,
$$

in case the d.f. $G(\cdot)$ is stable with exponent $0<\alpha<2$.

It is interesting to remark that this result may be partially verified by other means. BROECKX, GoOvAERTS and DE VyLDER (1984) show that, for any claim size distribution, $\psi(x)$ is subject to upper and lower bounds whose asymptotic forms are:

$$
\frac{1}{\eta}\left[1-G(x)+x^{-1} \int_{0}^{x} y d G(y)\right] \text { and } \frac{1}{\eta}[1-G(x)]
$$

respectively for large $x$.

These may be rewritten as:

$$
\text { const. } \times x^{-1} \int_{0}^{x}[1-G(y)] d y,
$$

and

$$
\text { const. } \times[1-G(x)]
$$

respectively for large $x$.

Now consider $G(\cdot)$ in the domain of attraction of a stable distribution with exponent $0<\alpha<1$.

By (5.4.3.4), the asymptotic forms of these bounds are both const. $\times x^{-\alpha}$ for large $x$; which verifies (5.4.3.8) in this case.

\subsubsection{Moderately Long Tailed Claim Size Distributions}

Section 5.2 considered short tailed claim size distributions, i.e., those whose m.g.f.'s converge.

Of those whose m.g.f.'s do not converge, essentially the subexponential family was considered in Section 5.3. As remarked by EMBREChTS and VeraverbekE (1982, p. 62), these distributions are sufficiently long tailed that the aggregate of $n$ claims is likely to be dominated by one very large claim. 
Sections 5.2 and 5.3 indicate the radically different behaviour of $\psi(\cdot)$ under the influence of these long and short tailed claim size distributions. It is perhaps of interest to consider "moderately long tailed" claim size distributions, i.e., those whose m.g.f.'s do not converge but are in some sense close to doing so.

\section{REFERENCES}

Bowers, N. L., Gerber, H. U., Hickman, J. C., Jones, D. A. and Nesbitt, C. J. (1982). Society of Actuaries Study Note on Risk Theory. Society of Actuaries: Chicago.

BRoECKX, F., GoovAerTS, M. and DE VyLDER, F. (1984). Ordering of Risks and Ruin Probabilities. Paper presented to the Macquarie University General Insurance Seminar, October 1984.

BühlmanN, H. (1970). Mathematical Methods in Risk Theory. Springer-Verlag: Berlin.

Bühlmañ, H., Gagliardi, B., Gerber, H. U. and STraub, E. (1977). Some Inequalities for Stop-Loss Premiums. Astin Bulletin 9, 75-83.

DE Vylder, F. (1978). A Practical Solution to the Problem of Infinite Time Ruin Probability. Scandinavian Actuarial Journal.

Embrechts, P. and Veraverbeke, N. (1982). Estimates for the Probability of Ruin with Special Emphasis on the Possibility of Large Claims. Insurance: Mathematics and Economics 1, 55-72.

Feller, W. (1966). An Introduction to Probability Theory and its Applications, Vol. II. 2nd ed. John Wiley \& Sons Inc.: New York.

Gerber, H. U. (1979). An Introduction to Mathematical Risk Theory. Monograph No. 8, S. S. Huebner Foundation for Insurance Education, Wharton School, University of Pennsylvania, Philadelphia. Distributed by Richard D. Irwin, Inc., Homewood, Illinois.

Goovaerts, M. and DE VyLDER, F. (1984a). A Stable Recursive Algorithm for Evaluation of Ultimate Ruin Probabilities. Astin Bulletin 14, 53-59.

Goovaerts, M. and DE VYLDeR, F. (1984b). Dangerous Distributions and Ruin Probabilities in the Classical Risk Model. Transactions of the 22nd International Congress of Actuaries 3, 111-120.

GoovaerTs, M., DE VyLder, F. and HAEZENDONCK, J. (1984). Stop-Loss Dominance. Blätter der Deutschen Gesellschaft für Versicherungsmathematik 16, 301-310.

Lundererg, F. (1909). Über die Theorie der Rückversicherung. Ber. VI. Intern. Kong. Versich. Wissens. 1, 877-948.

LUNDBERG, F. (1926-1928). Forsakringsteknisk Riskutjämning, I, Teori; II, Statistik. Englund: Stockholm.

PANJER, H. H. (1981). Recursive Evaluation of a Family of Compound Distributions. Astin Bulletin $12,22-26$.

Panjer, H. H. (1984). Direct Calculation of Ruin Probabilities. Submitted for publication.

SEAL, H. L. (1979). Stochastic Theory of a Risk Business. John Wiley \& Sons Inc.: New York.

SUNDT, B. and JEWELl, W. S. (1981). Further Results on Recursive Evaluation of Compound Distributions. Astin Bulletin 12, 27-39.

TAYLOR, G. C. (1974). On the Radius of Convergence of an Inverted Taylor Series with Particular Reference to the Solution of Characteristic Equations. Scandinavian Actuarial Journal 1974, 11-20.

TAYLOR, G. C. (1976). Use of Differential and Integral Inequalities to Bound Ruin and Queueing Probabilities. Scandinavian Actuarial Journal 1976, 197-208. 